\title{
A co-infection case report of Taenia saginata in a patient with subclinical clonorchiasis confirmed by the combination of diagnostic tools
}

Ju Hyeon Shin ${ }^{1}$, Eun Jeong Won ${ }^{2^{*}}$, Jee Seung Jung ${ }^{2}$, Kyung-Hwa Park ${ }^{3}$, Kwang $\|$ Nam$^{4}$, Soo Hyun Kim ${ }^{1}$ and Jong Hee Shin ${ }^{1}$

\begin{abstract}
Background: Clonorchiasis is the common parasitic infection in the general population of the Republic of Korea, however, taeniasis is scarcely reported recently. Here, we describe a case of co-infection with the cestode $T$. saginata in a patient with subclinical clonorchiasis diagnosed by a combination of diagnostic tools in Korea.

Case presentation: A 56-year-old man visited the hospital having passed proglottids in his stool for the past two months and brought a stool sample with segments to our hospital. He had no abdominal symptoms, such as nausea, vomiting, abdominal pain, diarrhea, or constipation. He used to consume raw beef and fish frequently. We could not find evidence of gravid proglottids which contain fully developed uteri filled with ova or branched uterine structures, within the submitted sample. To identify the tapeworm species, we carried out molecular analyses on the proglottids. The cox 1 and efla sequences had a 100\% match with those of T. saginata and differed from the sequences of the other Taenia species. Upon examination of stool samples fixed by formalin-ether concentration method, no Taenia species ova were observed in 10 slides. Instead, C. sinensis ova were observed, despite the level of lgG specific to C. sinensis being within the normal range. The patient was treated with praziquantel $(25 \mathrm{mg} / \mathrm{kg}$, three times a day) for 3 days, and subsequently $C$. sinensis ova were not found in his stool.
\end{abstract}

Conclusion: Our case indicates that a combination of morphological, serological, and molecular diagnostic tools should be used for the accurate diagnosis of subclinical parasitic infections.

Keywords: Taenia saginata, Clonorchis sinensis, Molecular diagnosis, Ova, ELISA

\section{Background}

Intestinal parasitic infections are still a major public health issue in worldwide [1]. Until the 1970s, many Koreans had intestinal parasitic infections, mostly from soil-transmitted helminthes [2]. Although overall helminth egg-positive rate was $84.3 \%$ in the 1st Nationwide Survey [2], a dramatic decline of the egg-positive rate has been shown to $2.6 \%$ in the 8th Nationwide Survey, 2013 [3]. Thus, at present, intestinal parasitic infections are not recognized as a critical problem in Korea. The public health focus has also shifted

\footnotetext{
* Correspondence: Parasite.woni@jnu.ac.kr

${ }^{2}$ Department of Parasitology and Tropical Medicine, Chonnam National University Medical School, 264, Seoyang-ro, Hwasun-eup, Hwasun-gun, Jeollanam-do 58128, Republic of Korea

Full list of author information is available at the end of the article
}

from soil-transmitted helminthiases to food-borne parasitic infections. Of food-borne parasites, Clonorchis sinensis showed the highest prevalence in Korea showing 2.4\% of egg-positive rate in general population [3]. The parasite is more prevalent in populations living by rivers, with an egg-positive rate of $11.4 \%$ [4], as humans usually become infected after ingesting raw or undercooked freshwater fish harboring C. sinensis metacercariae [5]. Human taeniasis, another food-borne parasitic infection, is a zoonotic disease, because it involves pigs (Taenia solium and Taenia asiatica) or cattle (Taenia saginata) as an intermediate host and humans as the definitive host [6, 7]. Studies dating back to 1924 reported Taenia prevalence in Korea at $7.2 \%$ or higher [8-10], but the national prevalence was $1.98 \%$ in 1971 [2].

(c) The Author(s). 2019 Open Access This article is distributed under the terms of the Creative Commons Attribution 4.0 International License (http://creativecommons.org/licenses/by/4.0/), which permits unrestricted use, distribution, and 
The national prevalence continued to decline, and was reported as $0.004 \%$ in 2013 [3]. Here, we describe a case of co-infection case with the cestode $T$. saginata and the trematode $C$. sinensis by using a combination of diagnostic tools in a patient with subclinical parasitic infection.

\section{Case presentation}

A 56-year-old man complained of passing proglottids in his stool intermittently over the last two months. No abdominal symptoms, such as nausea, vomiting, abdominal pain, diarrhea, or constipation were present. He reported frequent consumption of raw beef and fish (both marine and freshwater fish), and had no history of traveling abroad. He had previously obtained $400 \mathrm{mg}$ of albendazole from the pharmacy and taken it once orally without clinical improvement. After that, he was prescribed 600 mg of praziquantel at a local clinic and had taken it once orally as well. He brought his stool sample, which included the passed segments to our hospital (Fig. 1a). The segments were pressed between two microscope slides and examined macroscopically without staining. We could not observe gravid proglottids, which contain fully developed uteri filled with ova, or branched uterine structures. To identify the tapeworm species, we conducted molecular analysis using the proglottid segments. Genomic DNA was extracted using the QIAamp DNA Mini Kit (Qiagen, Hilden, Germany) and subsequently used as a template for polymerase chain reaction (PCR). The mitochondrial cytochrome $c$ oxidase subunit I ( $\operatorname{cox} 1)$ gene and partial sequences of elongation factor-1 alpha (efla) were targeted for PCR amplification. The sequences of the PCR primers used were: T1F (5'-ATATTTACT TTAGATCAT AAGCGG-3') and T1R (5'-ACGAGAAAATATATTAG TCATAAA-3') for cox1, and Tae_ef1/F4 (5'-TGTGGT GGAATCGATAAAAGG-3') and Tae_ef1/R4 (5'-TCGA TCTCATGTCACGAACG -3') for ef1a [11, 12]. PCR was carried out using a $30 \mu \mathrm{L}$ reaction mixture containing $15 \mu \mathrm{L}$ Smart $2 \times$ PCR Pre-Mix (SolGent Co., Ltd., Daejeon, Korea), $2 \mu \mathrm{L}$ template DNA, $10 \mu \mathrm{M}$ of each primer, and $11 \mu \mathrm{L}$ distilled water, as described in a previous study [13]. The amplification process comprised 35 cycles of denaturation $\left(94{ }^{\circ} \mathrm{C}\right.$ for $\left.30 \mathrm{~s}\right)$, annealing $\left(60^{\circ} \mathrm{C}\right.$ for $\left.30 \mathrm{~s}\right)$, and extension $\left(72^{\circ} \mathrm{C}\right.$ for $\left.80-90 \mathrm{~s}\right)$. The PCR products were sent to Macrogen (Korea) for direct sequencing using the same PCR primers. The 480-bp cox1 sequence had a $100 \%$ match with the $T$. saginata sequence and a $94.8 \%$ match with the T. asiatica sequence. The 1078-bp ef1a sequence also had a $100 \%$ match with the sequence of $T$. saginata, a 99.3\% match with the sequence of T. asiatica and a 95.3\% match with the sequence of T. solium. The three Taenia

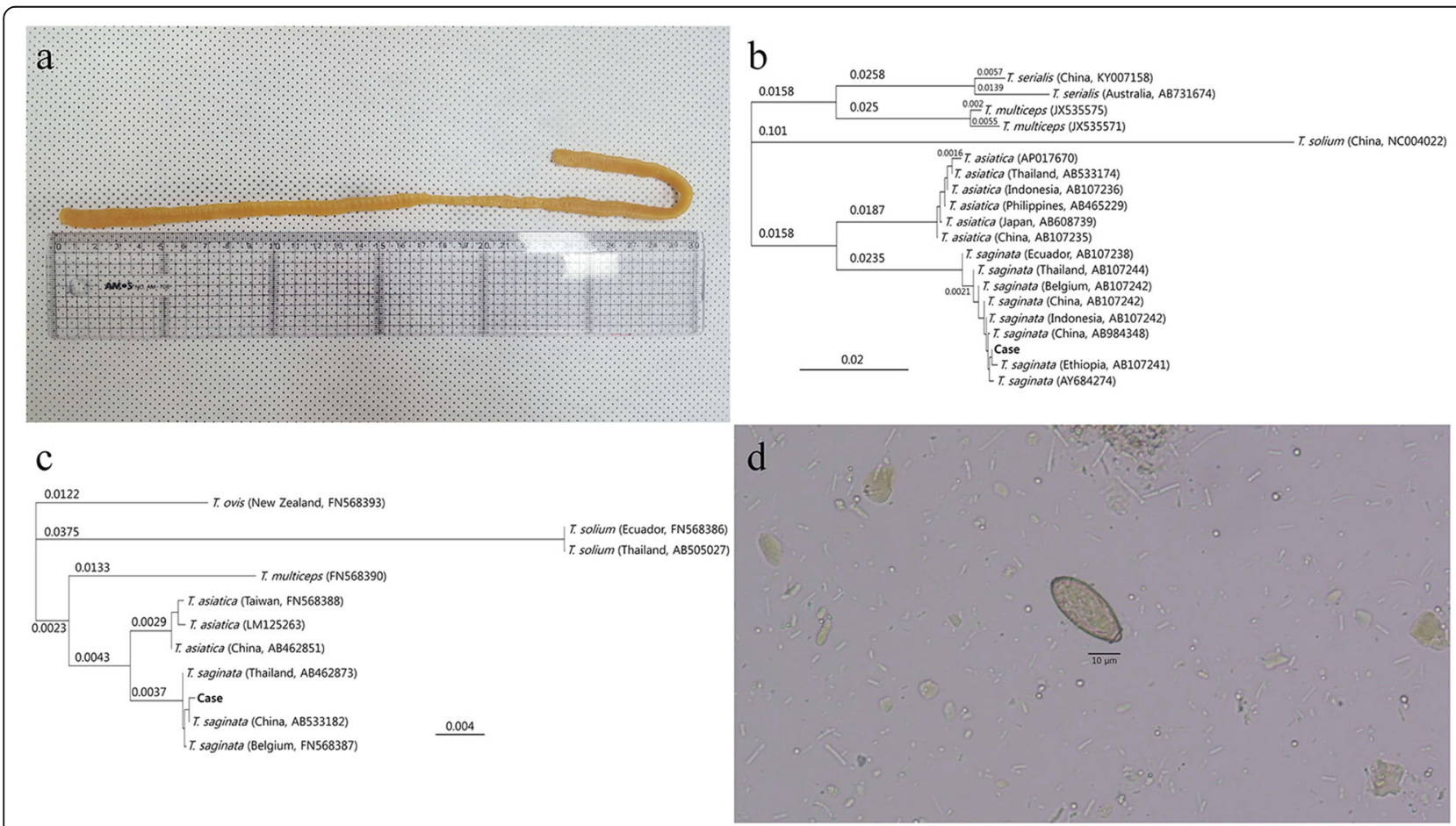

Fig. 1 a Overall appearance of proglottids in the patient's feces, about $37 \mathrm{~cm}$ in length. b Phylogenetic tree for the cox1 gene and (c) ef1a gene obtained from the patient's Taenia tapeworm, compared to other Taenia species in GenBank. The GenBank accession number is indicated. The scale bar indicates a sequence distance of 0.02 and 0.004 nucleotide substitutions per site. $\mathbf{d}$ Ovum of $C$. sinensis found in the patient's stool specimen. A scale bar is included 
species that infect humans had nucleotide differences at 73 and 57 polymorphic sites for the $\operatorname{cox} 1$ and efla sequences, respectively (Additional file 1). Of these polymorphic sites, three sites (nucleotides 294, 336, 405) in $\operatorname{cox} 1$ differentiated the three species. DNA sequences were aligned using the CLUSTAL W computer program [14]. Phylogenetic trees were constructed using the neighbor joining method [15] and genetic distances were computed using the Tamura-Nei method using the Geneious computer program (Fig. 1b and c). The neighbor joining tree used GenBank sequences derived from samples collected in Asia, and indicated that our specimen was in the same phylogenetic group as $T$. saginata, but not in the same group as T. asiatica or T. solium. In addition to molecular analysis, we also examined the patient's stool specimen using the formalin-ether concentration method. However, we could not observe any Taenia species ova on the 10 slides examined. Ova were not observed inside the proglottids either, indicating that these proglottids were immature. Instead, $C$. sinensis ova were observed on one slide (Fig. 1d). Conversely, the level of serum IgG specific to $C$. sinensis measured using Enzyme-Linked Immunosorbent Assay (ELISA) was within the normal range. He was treated with praziquantel at the recommended dose of $25 \mathrm{mg} / \mathrm{kg}$ three times daily for 3 days [16]. After treatment, no proglottids or ova of $C$. sinensis were found in his stool.

\section{Discussion and conclusions}

Human taeniasis is usually diagnosed by observing ova or gravid proglottids in the patient's stool. From diagnoses conducted after 1993, the Taenia tapeworms infecting humans in Korea were identified as T. solium, T. saginata, and $T$. asiatica $[5,17,18]$. However, additional differential modalities may be required to clearly distinguish among these species, as morphological characteristics, such as the presence of an unarmed rostellum on the scolex of adult, the large number of uterine twigs, and the presence of a posterior protuberance, can be difficult to observe in individual strobili $[17,18]$. Directe sequencing of $\operatorname{cox} 1$ sequences have been used to identify the species causing human taeniasis. Cho et al. successfully used cox 1 to distinguish between T. saginata and T. asiatica [11]. Recently, studies conducted in China and Lao People's Democratic Republic reported that hybridization between the three Taenia species cab iccyr, based on sequencing nuclear and mitochondrial genes [19, 20]. In our case, as we did not observe uterine structures or ova in the proglottids in our patient's stool sample, these proglottids were likely immature. As such, we were unable to identify the tapeworm to the species-level using morphological characters, and thus sequenced both the nuclear gene, efla, and the mitochondrial gene, coxl, from the proglottid sample as well. Matches at several polymorphic sites confirmed that the Taenia specimen in this case is most likely $T$. saginata, and closely related to $T$. saginata, specimens sampled previously in Korea, China, Indonesia, Thailand and other countries (Fig. 1b and c). Here, we diagnosed that this case of human taeniasis was caused by T. saginata. We confirmed that tapeworm species by sequencing nuclear and mitochondrial gens, which successfully differentiated $T$. saginata from other Taenia species. Notably, we discovered that co-existence of taeniasis in a patient with subclinical clonorchiasis using a combination of diagnostic tools. Even in the era of molecular tools, the expertise in microscopy is still essential to achieve a correct diagnosis. Further, subclinical clonorchiasis in this case was confirmed only by examining the stool sample using microscopy. Regarding the use of ELISA to diagnose the presence of $C$. sinensis, ELISA results should be interpreated with caution, as the result can be negative even with an egg-positive [21, 22].

Early documentation of human taeniasis in Korea reported that the prevalence of Taenia was ranging from 7.2 to $12.0 \%$ [8-10]. More recently (2004-2008), the prevalence had declined to low levels of $0-0.01 \%[3,23]$. Since 2008, there has only been one study that documented four cases of $T$. saginata, which were diagnosed by sequencing of $\operatorname{cox} 1$ [11]. Some researchers have suggested that human taeniasis is now close to absent in Korea, but cases of infection may be hidden, especially in areas where taeniasis was previously ubiguitous. In our case, the patient was from Jeollanam-do, the province with the second highest prevalence of taeniasis after Jeju-do (Island) according to the 1st Nationwide Survey [2]. Co-infection with other parasites can frequently occur in areas with high parasite prevalence, even though the helminthes do not share the same intermediate host [16]. Moreover, people who enjoy eating both raw beef and freshwater fish, as with our patient, have a higher chance of co-infection. The habit of eating raw fish or beef is deeply rooted in traditional customs among residents of rural areas of Korea [4]. In general, educating the public about not eating raw beef and freshwater fish is important for reducing the incidence of food-borne parasitic infections in Korea.

Initially, the patient took albendazole obtained from a pharmacy instead of visiting a hospital, although the medication was not effective. This may be due in part to the National Deworming Campaign in Korea over the past years that focused on soil-transmitted helminthiases and highlighted repeated administration of albendazole to control parasitic infections. An initial 600-mg tablet of praziquantel $(10 \mathrm{mg} / \mathrm{kg})$ may be sufficient for treating taeniasis in this case. The patient also received additional praziquantel at a dosage of $75 \mathrm{mg} / \mathrm{kg} /$ day for 3 days to treat clonorchiasis. Although we could not find scolex of Taenia species in the submitted specimens, the treatment may have been effective in controlling both taeniasis and clonorchiasis. 
In this study, we described an unusual case of co-infection of $T$. saginata in a patient with subclinical clonorchiasis in Korea. Our results indicate that diagnosis through molecular methods may be helpful in cases with ambiguous morphological characters such as immature proglottids. Overall, a combination of diagnostic tools should be used for the accurate diagnosis of subclinical parasitic infections.

\section{Additional file}

Additional file 1: Polymorphic sites of $\operatorname{cox} 1$ and ef1a sequences of the submitted proglottids compared with those of human Taenia tapeworms. Numbers indicate the positions from $5^{\prime}$ end of the cox 1 or efla gene, and dots indicate sequence matches with $T$. saginata. ${ }^{*}$ Three sites (nucleotide $294,336,405$ ) were distinguishable substitiutions in all of three human Taenia tapeworms. (XLSX $16 \mathrm{~kb})$

\section{Abbreviations}

cox1: Cytochrome c oxidase subunit l; ef1a: Elongation factor-1 alpha; ELISA: Enzyme-linked immunosorbent assay; PCR: Polymerase chain reaction

\section{Acknowledgements}

Not applicable.

\section{Funding}

No funding was required for the writing of this case report.

\section{Availability of data and materials}

Not applicable (no datasets were analyzed or generated during this study).

\begin{abstract}
Authors' contributions
KHP participated in the clinical care of the patient. JSJ performed experiments. $\mathrm{KIN}$, SHK and JHS1 advised about and supervised the experiments and interpretations. JHS2 and EJW wrote the manuscript.EJW was responsible for the concept of and critical contribution and revision to this manuscript. JHS1 corresponding to JHS and JHS2 corresponding to JHS. All authors critically reviewed the manuscript for publication. All authors read and approved the final manuscript.
\end{abstract}

\section{Ethics approval and consent to participate}

The collection of fecal samples including tapeworm conducted in accordance with the guidelines and approval of the Institutional Review Board of Chonnam National University Hospital (IRB CNUH-2015-052) and all data analyzed were anonymized. No information was used that could lead to patient identification.

\section{Consent for publication}

Written consent to publish this case report was obtained from the patient (Consent No. 2018-P02).

\section{Competing interest}

The authors declare that they have no competing interests.

\section{Publisher's Note}

Springer Nature remains neutral with regard to jurisdictional claims in published maps and institutional affiliations.

\section{Author details}

'Department of Laboratory Medicine, Chonnam National University Hospital, Gwangju, Republic of Korea. ${ }^{2}$ Department of Parasitology and Tropical Medicine, Chonnam National University Medical School, 264, Seoyang-ro, Hwasun-eup, Hwasun-gun, Jeollanam-do 58128, Republic of Korea. ${ }^{3}$ Department of Infectious Diseases, Chonnam National University Hospital, Gwangju, Republic of Korea. ${ }^{4}$ Department of Anatomy, Chonnam National University Medical School, Hwasun-gun, Jeollanam-do, Republic of Korea.
Received: 2 September 2018 Accepted: 29 January 2019

Published online: 06 February 2019

\section{References}

1. Hotez PJ, Brindley PJ, Bethony JM, King CH, Pearce EJ, Jacobson J. Helminth infections: the great neglected tropical diseases. J Clin Invest. 2008;118: $1311-21$.

2. Ministry of Health and Social Affairs and Korean Association for Parasite Eradication. Prevalence of intestinal parasitic infections in Korea. The 1th report. Seoul, Korea. 1971. 1-26. (in Korean).

3. Korea Centers for Disease Control and Prevention. National survey of intestinal parasitic infections in Korea, 8th report 2013. KCDC 2014;1:89-94. (in Korean).

4. Cho SH, Lee KY, Lee BC, Cho PY, Cheun HI, Hong ST, Sohn WM, Kim TS. Prevalence of Clonorchiasis in southern endemic areas of Korea in 2006. Korean J Parasitol. 2008;46:133-7.

5. Cho PY, Na BK, Choi KM, Kim JS, Cho SH, Lee WJ, Lim SB, Cha SH, Park YK, Pak JH, Lee HW, Hong SJ, Kim TS. Development of a polymerase chain reaction applicable to rapid and sensitive detection of Clonorchis sinensis eggs in human stool samples. Pathog Glob Health. 2013;107:253-9.

6. Chai JY. Human taeniasis in the Republic of Korea: hidden or gone? Korean J Parasitol. 2013:51:9-17.

7. Dorny P, Praet N. Taenia saginata in Europe. Vet Parasitol. 2007;149:22-4.

8. Kobayashi H, Kwon N. Studies on the intestinal parasites of Koreans: first report. J Chosen Med Ass. 1917;19(Suppl):72-8 (in Japanese).

9. Kojima R, Ko T. Researches on intestinal parasites of Korea in south Gyeongsang-do, especially on the distribution of the liver fluke. J Chosen Med Ass. 1919;26:42-86 (in Japanese).

10. Uchida R. Results of fecal examination on intestinal helminths among Korean prisoners in the Seodaemoon jail. J Chosen Med Ass. 1924;46: 74-83 (in Japanese).

11. Cho J, Jung BK, Lim H, Kim MJ, Yooyen T, Lee D, Eom KS, Shin EH, Chai JY. Four cases of Taenia saginata infection with an analysis of COX1 gene. Korean J Parasitol. 2014;52:79-83.

12. Okamoto M, Nakao M, Blair D, Anantaphruti MT, Waikagul J, Ito A. Evidence of hybridization between Taenia saginata and Taenia asiatica. Parasitol Int. 2010;59:70-4.

13. Jeon HK, Kim KH, Eom KS. Molecular identification of Taenia specimens after long-term preservation in formalin. Parasitol Int. 2011:60:203-5.

14. Thompson JD, Higgins DG, Gibson TJ. CLUSTAL W: improving the sensitivity of progressive multiple sequence alignment through sequence weighting, position-specific gap penalties and weight matrix choice. Nucleic Acids Res. 1994:22:4673-80.

15. Saitou N, Nei M. The neighbor-joining method: a new method for reconstructing phylogenetic trees. Mol Biol Evol. 1987;4:406-25.

16. Hong ST, Rim HJ, Min DY, Li X, Xu J, Feng Z, Lee SH. Control of clonorchiasis by repeated treatments with praziquantel. Korean J Parasitol. 2001;39:285-92.

17. Eom KS, Rim HJ. Morphologic descriptions of Taenia asiatica sp. n. Korean J Parasitol. 1993;31:1-6.

18. Eom KS, Rim HJ. Epidemiological understanding of Taenia tapeworm infections with special reference to Taenia asiatica in Korea. Korean J Parasitol. 2001;39:267-83.

19. Yamane K, Suzuki Y, Tachi E, Li T, Chen X, Nakao M, Nkouawa A, Yanagida T, Sako Y, Ito A, Sato H, Okamoto M. Recent hybridization between Taenia asiatica and Taenia saginata. Parasitol Int. 2012:61:351-5.

20. Sato MO, Sato M, Yanagida T, Waikagul J, Pongvongsa T, Sako Y, Sanguankiat S, Yoonuan T, Kounnavang S, Kawai S, Ito A, Okamoto M, Moj K. Taenia solium, Taenia saginata, Taenia asiatica, their hybrids and other helminthic infections occurring in a neglected tropical diseases' highly endemic area in Lao PDR. PLoS Negl Trop Dis. 2018;12:e0006260.

21. Kim YJ, Lee SM, Choi GE, Hwang SH, Kim HH, Lee EY, Chang CL. Performance of an enzyme-linked immunosorbent assay for detection of Clonorchis sinensis infestation in high- and low-risk groups. J Clin Microbiol. 2010:48:2365-7.

22. Han S, Zhang $X$, Wen J, Li Y, Shu J, Ling H, Zhang F. A combination of the Kato-Katz methods and ELISA to improve the diagnosis of clonorchiasis in an endemic area. China PLoS One. 2012;7:e46977.

23. Chai JY, Park JH, Guk SM, Kim HJ, Kim WH, Kim JL, Gu YS, Shin EH, Park HM, Hong KS, Kim SD, Lee SH. Status of intestinal parasite infections among 4,137 residents from provinces nationwide and metropolitan areas in the Republic of Korea (2004). Infect Chemother. 2006;38:198-203. 